\title{
The Acoustic Analogy - A Powerful Tool in Aeroacoustics with Emphasis on Jet Noise Prediction
}

\author{
F. Farassat ${ }^{*}$, Michael J. Doty ${ }^{\dagger}$, and Craig A. Hunter \\ NASA Langley Research Center, Hampton, VA, 23681
}

\begin{abstract}
The acoustic analogy introduced by Lighthill to study jet noise is now over 50 years old. In the present paper, Lighthill's Acoustic Analogy is revisited together with a brief evaluation of the state-of-the-art of the subject and an exploration of the possibility of further improvements in jet noise prediction from analytical methods, computational fluid dynamics (CFD) predictions, and measurement techniques. Experimental Particle Image Velocimetry (PIV) data is used both to evaluate turbulent statistics from Reynolds-averaged Navier-Stokes (RANS) CFD and to propose correlation models for the Lighthill stress tensor. The NASA Langley Jet3D code is used to study the effect of these models on jet noise prediction. From the analytical investigation, a retarded time correction is shown that improves, by approximately $8 \mathrm{~dB}$, the over-prediction of aft-arc jet noise by Jet3D. In experimental investigation, the PIV data agree well with the CFD mean flow predictions, with room for improvement in Reynolds stress predictions. Initial modifications, suggested by the PIV data, to the form of the Jet3D correlation model showed no noticeable improvements in jet noise prediction.
\end{abstract}

\section{Nomenclature}

$\alpha_{L}$

$c_{\infty}$

$C=\left|1+M_{c} \cos \theta\right|$

$C_{\infty}=\left|1+M_{\infty} \cos \theta\right|$

$D_{c}$

$\varepsilon$

$k$

$l_{1}, l_{2}, l_{3}$

$L$

$L_{i i}$

$M_{c}$

$M_{\infty}$

$\mu$

$N P R$

$p$

$Q$

$\theta$

$\theta *$

$\vec{r}$

$r$

$\hat{r}$

$\vec{r} *$

$r^{*}$
Length scale calibration constant

Ambient speed of sound ( $\mathrm{m} / \mathrm{s}$ )

Local source convection factor

Flight convection factor

Core nozzle diameter $(=0.128 \mathrm{~m})$

Dissipation rate of $k\left(\mathrm{~m}^{2} / \mathrm{s}^{3}\right)$

Turbulent kinetic energy per unit mass $\left(\mathrm{m}^{2} / \mathrm{s}^{2}\right)$

Turbulence length scales (m)

Differential operator

Experimental integral length scale

Source convection Mach number

Aircraft Mach number

Correlation calibration constant

Nozzle Pressure Ratio

Fluctuating acoustic pressure $\left(\mathrm{N} / \mathrm{m}^{2}\right)$

Differential operator

Observer angle from jet axis (deg)

Geometric observer angle from jet axis (deg)

Acoustic radius vector (m) at retarded time, $\vec{r}=\vec{x}-\vec{z}$

Magnitude of $\vec{r}$ (m)

Acoustic radius unit vector, $\hat{r}=\vec{r} / r$

Geometric radius vector $(\mathrm{m})$

Magnitude of $\vec{r} *(\mathrm{~m})$

\footnotetext{
* Aerospace Engineer, Aeroacoustics Branch, Mail Stop 461, Associate Fellow AIAA.

${ }^{\dagger}$ National Research Council Postdoctoral Researcher, Mail Stop 166, Senior Member AIAA.

* Aerospace Engineer, Configuration Aerodynamics Branch, Mail Stop 499.
} 


$R_{i m}$
$\rho$
$\rho_{i j}$
$T_{i j}, T{ }_{k l}{ }$
$T_{o}$
$\tau$
$\tilde{\tau}$
$\vec{u}$
$\vec{V}$
$\vec{v}$
$\left\langle v_{i} v_{m}\right\rangle$
$\left\langle v_{i} v_{m}^{\prime}\right\rangle$
$\vec{x}$
$x$
$\xi$
$\vec{z}$
$\vec{\zeta}$

\author{
Spatial Correlation Function $\left(\mathrm{m}^{2} / \mathrm{s}^{2}\right)$ \\ Density $\left(\mathrm{kg} / \mathrm{m}^{3}\right)$ \\ Correlation coefficient function \\ Lighthill stress tensor $\left(\mathrm{N} / \mathrm{m}^{2}\right)$ \\ Stagnation Temperature (K) \\ Time delay in observer frame (s) \\ Moving frame time delay (s), $\tilde{\tau}=\tau / C$ \\ Total velocity $(\mathrm{m} / \mathrm{s}), \vec{u}(\vec{z}, t)=\vec{V}(\vec{z})+\vec{v}(\vec{z}, t)$ \\ Mean Flow Velocity $(\mathrm{m} / \mathrm{s}), \vec{V}=\vec{V}(\vec{z})$ \\ Turbulent velocity $(\mathrm{m} / \mathrm{s}), \vec{v}=\vec{v}(\vec{z}, t)$ \\ One-point turb. velocity correlation $\left(\mathrm{m}^{2} / \mathrm{s}^{2}\right)$ \\ Two-point turb. velocity correlation $\left(\mathrm{m}^{2} / \mathrm{s}^{2}\right)$ \\ Fixed observer position vector (m) \\ Jet axis coordinate $(\mathrm{m})$ \\ Separation distance \\ Moving frame source position vector (m) \\ Moving frame separation vector (m)
}

\section{Introduction}

$T_{\mathrm{t}}^{\mathrm{h}}$

he acoustic analogy (AA) introduced by Lighthill to study jet noise is now over 50 years old ${ }^{1}$. In addition to Lighthill, other researchers such Ribner ${ }^{2}$, Lilley ${ }^{3}$, Curle ${ }^{4}$, and Ffowcs Williams ${ }^{5}$ have made significant contributions to the theory and applications of AA. Half a century of work on AA has established this theory firmly in the minds and hearts of most aeroacousticians. It can be argued that AA has been most successful in predicting rotating blade noise based on the Ffowcs Williams-Hawkings (FW-H) equation ${ }^{6}$. The main reason for this success is that the acoustic sources are generally deterministic and are distributed on the blade surface. The deterministic character of the acoustic sources has made it possible to use advanced CFD techniques to supply the input data needed for computation of the noise of rotating machinery. Applications of AA to jet noise prediction have been less successful primarily because we are not able to fully supply the turbulence data needed for the job. But this is not the sole problem. It is, therefore, time to look once more at AA and highlight some factors responsible for the success of the rotating blade noise prediction which may be useful in jet noise analysis.

In his keynote address at the 2003 AIAA/CEAS Aeroacoustics Conference in Hilton Head, South Carolina, Geoffrey Lilley spoke of the need for "close cooperation between theoretical and experimental teams since in my mind that cooperation is essential to generate successful noise reduction schemes and of course to calibrate any theoretical predictions." ${ }^{7}$ The present paper is the result of one such collaboration between researchers with experimental, theoretical, computational backgrounds. In this paper, we briefly evaluate the state-of-the art of AA and explore further improvements in noise prediction in light of the availability of advanced analytical, computational fluid dynamics (CFD), and measurement techniques.

In the next section we define broadly what the acoustic analogy is. This leads us to the definition of an acoustic source. We distinguish the literal and mathematical definitions of an acoustic source. We argue that the usefulness of AA can be extended further by inclusion of near field terms and derivation of exact analytical results that utilize advanced flow simulation. We propose one correction to the AA involving the inclusion of the forward flight effect in the emission time calculation. In the remainder of this paper, we explore several different models of crosscorrelation of the Lighthill stress tensor based on PIV data. The conclusions follow.

\section{The Acoustic Analogy (AA)}

We start by giving a general definition of the acoustic analogy (AA) and defining the meaning of an acoustic source, with the focus on predicting noise from turbulence and surfaces in motion confined to a finite region of space $\mathbf{V}$. We assume that we have the information about all flow parameters and surface pressure distributions 
inside $\mathbf{V}$ for a sufficiently long period of time, and that pressure fluctuations outside the volume $\mathbf{V}$ satisfy the linear wave equation. Suppose the conservation laws can be manipulated into the following form:

$$
L p=Q
$$

where $L$ is a linear or nonlinear partial differential operator such that outside $\mathbf{V}$ it becomes the linear wave operator. Here, $p$ is the acoustic pressure, or $\rho c_{\infty}^{2}$, where $\rho$ is the density perturbation and $c_{\infty}$ is the speed of sound outside the volume $\mathbf{V}$. The inhomogeneous term of this differential operator, $Q$, depends on the flow parameters and is assumed known. We will define the acoustic analogy as any noise prediction methodology based on eq. (1). By this definition, all noise prediction methodologies using the Lighthill's equation ${ }^{1}$, the Ffowcs Williams-Hawkings $(\mathrm{FW}-\mathrm{H})$ equation ${ }^{6}$, Lilley's equation ${ }^{3}$, or Ribner's equation ${ }^{2}$ are in fact based on AA.

In practice, the main purpose of using AA in noise prediction is to speed up the process of getting numerical results. Obtaining the flow information inside $\mathbf{V}$ is, in general, expensive either using experiments or CFD. Therefore, it is advantageous to make the volume $\mathbf{V}$ as small as possible. Solving the linear or nonlinear differential operator of eq. (1) is much cheaper, particularly when dealing with a linear differential operator such as the wave operator where Green's function method can be utilized. We can extend the definition of the AA to include any noise prediction methodology that essentially uses two different approaches to obtain flow field information in the near field and the acoustic information in the far field.

We will now address the question of "What is an acoustic source?". There seem to be two distinct answers to this question, one literal and one mathematical, and the two are sometimes confused. When we say that a truck traveling on a highway is the source of sound, we all understand that the engine, tires and perhaps the flow of air over the body of the truck are generating noise. These make up the literal definition. The mathematical definition of the source of sound depends on the way we choose to formulate the problem. We propose to call the inhomogeneous source term $Q$ of eq. (1) the "mathematical" source of sound although we often choose to drop the adjective mathematical. Lighthill was careful to call quadrupole sources on the right side of his jet noise equation "fictitious". He was clearly implying that these quadrupoles are what we have called mathematical acoustic sources.

One can use physical arguments about noise generation, as Lighthill did so masterfully, but it is extremely important not to confuse physical arguments with mathematical reasoning. For instance, we know that sound rays bend in a moving medium with density and velocity gradients. In Lighthill's theory, the medium of propagation is quiescent; thus, there is no bending of sound rays from each volume source in the jet shear layers. This means that all rays emanating from a quadrupole source are straight lines. In this particular situation, the use of physical reasoning to account for the bending of rays becomes very difficult, and it may be advantageous to suppress one's intuition and to rely on mathematics instead. Mathematically, the magnitude and phasing of the quadrupole source distribution in AA account for the physical bending of rays in the region of the jet with density and velocity gradients. The best example demonstrating this effect of which the authors are aware is in the case of high-speed helicopter rotor noise prediction ${ }^{8}$. This problem is tractable numerically because of the deterministic nature of the quadrupole sources that can be obtained from computational fluid dynamics (CFD). It was shown that AA gives a waveform similar to that obtained from the fine resolution CFD with the correct steepening and level, and, thus, accounting for the refraction of sound in the regions of high velocity and density gradients.

For many years, AA was used to obtain qualitative jet noise prediction results such as those obtained by Lighthill $^{1}$, Ffowcs Williams ${ }^{5}$, Lilley ${ }^{3}$ and Ribner ${ }^{2}$. If quantitative results were desired, one had to accept input data that were often of dubious quality because of the lack of good experimental data or numerical simulations for the complex flow parameters of interest. The availability of high-fidelity Reynolds-averaged Navier-Stokes (RANS) computations has improved the situation dramatically, and hybrid methods involving RANS and Large Eddy Simulation (LES) will have practical application to complex jet flows within the next few years'. Because of these advances, it is time to change the attitudes, goals, and procedures used in jet noise prediction. Such a change has already occurred in other areas of aeroacoustics such as helicopter rotor and propeller noise prediction, where the state-of-the-art is highly advanced ${ }^{10}$. Can we learn any lessons from the success of rotating blade noise prediction? The answer is affirmative. We believe these lessons should be applied to jet noise prediction. We are interested in 
the development of sophisticated jet noise prediction codes that can handle complicated nozzle designs with the engine pylon extended into the jet stream. We, therefore, recommend that

i. As much as possible, one should strive to derive exact analytical results that include near field terms and flight effects,

ii. One should consider using new statistical parameters which can be obtained from CFD or experimental data.

Both these recommendations fall in the category of thinking outside of the box. We feel that Lighthill's AA, because of the simplicity of the Green's function of the wave equation, is highly suitable for obtaining exact analytical results $^{10,11}$. In the case of jet noise prediction, use of a cross-correlation function of the Lighthill stress tensor for two points in the frame moving with the jet ${ }^{11}$ should be further explored. Such a quantity can be both measured by the PIV technique and from CFD turbulence simulation. In the remainder of the paper, we work with the Lighthill AA. We work with the far field solution of Lighthill's equation in the form derived by Ffowcs Williams ${ }^{5}$. We show that, in the case of a jet in forward flight, the exact inclusion of emission times of quadrupole sources can improve acoustic prediction at angles which previously gave large errors compared to measured data.

In this paper, we use the NASA Langley Jet3D code ${ }^{12,13}$ to explore various aspects of jet noise prediction. As part of this, experimental PIV data is used to propose models of cross-correlation of the Lighthill stress tensor and study their effect on jet noise. The formulation of Lighthill's Acoustic Analogy (LAA) used in Jet3D was derived by Ffowcs Williams ${ }^{5}$ and is shown in equation 2, where the mean-square acoustic pressure is given as a function of observer location $\vec{x}$ and time delay $\tau$ :

$$
\left\langle p^{2}\right\rangle(\vec{x}, \tau)=\frac{1}{16 \pi^{2} c_{\infty}^{4}} \int_{J E T} \frac{\hat{r}_{i} \hat{r}_{j} \hat{r}_{m} \hat{r}_{n}}{C_{\infty} C^{5} r^{2}} \int_{-\infty}^{\infty} \frac{\partial^{4}}{\partial \tilde{\tau}^{4}}\left\langle T_{i j} T_{m n}^{\prime}\right\rangle d \vec{\xi} d \vec{z} .
$$

Here, $T_{i j}$ is the Lighthill Stress Tensor and all quantities inside the integrals are to be evaluated at a retarded time and corresponding position. The conventions in this formulation are shown in Figure 1, where effects of both aircraft motion (at Mach number $M_{\infty}$ ) and source convection (at Mach number $M_{c}$ ) are included. All Mach numbers are relative to the fixed observer, and are based on the ambient speed of sound $c_{\infty}$. It is important to note the relationship between source motion and convection; because acoustic sources in a jet (essentially convecting eddies) have a short lifetime, they possess a convective effect and Doppler shift that goes according to the convection Mach number $M_{c}$ but retarded time effects come in mainly through motion of the aircraft. Thus, the retarded time position of sources depends primarily on the flight Mach number $M_{\infty}$. Following the illustration in Figure 1, the geometric observer radius $r^{*}$ and geometric observer angle $\theta^{*}$ can be used to give the effective acoustic radius and observer angle at the retarded time (results shown assume subsonic $M_{\infty}$ ):

$$
\begin{gathered}
r=\frac{-M_{\infty} r^{*} \cos \theta^{*}+\sqrt{M_{\infty}^{2}\left(r^{*} \cos \theta^{*}\right)^{2}+\left(1-M_{\infty}^{2}\right) r^{2}}}{\left(1-M_{\infty}^{2}\right)}, \\
\theta=\cos ^{-1}\left[\frac{r^{*}}{r} \cos \theta^{*}-M_{\infty}\right] .
\end{gathered}
$$

\section{Application of Analytical Results}

The importance of properly accounting for retarded time will be demonstrated by looking at noise prediction for a bypass ratio (BPR) five core/fan nozzle (see Figure 2) with simulated forward flight. Noise prediction results for this nozzle, along with several others, were presented in detail in an earlier publication ${ }^{13}$ without retarded time correction. The nozzle is operating at a takeoff point with core conditions of $T_{o}=828 \mathrm{~K}$ and $N P R=1.56$, fan conditions of $T_{o}=350 \mathrm{~K}$ and $N P R=1.75$, and $M=0.28$ simulated forward flight. 


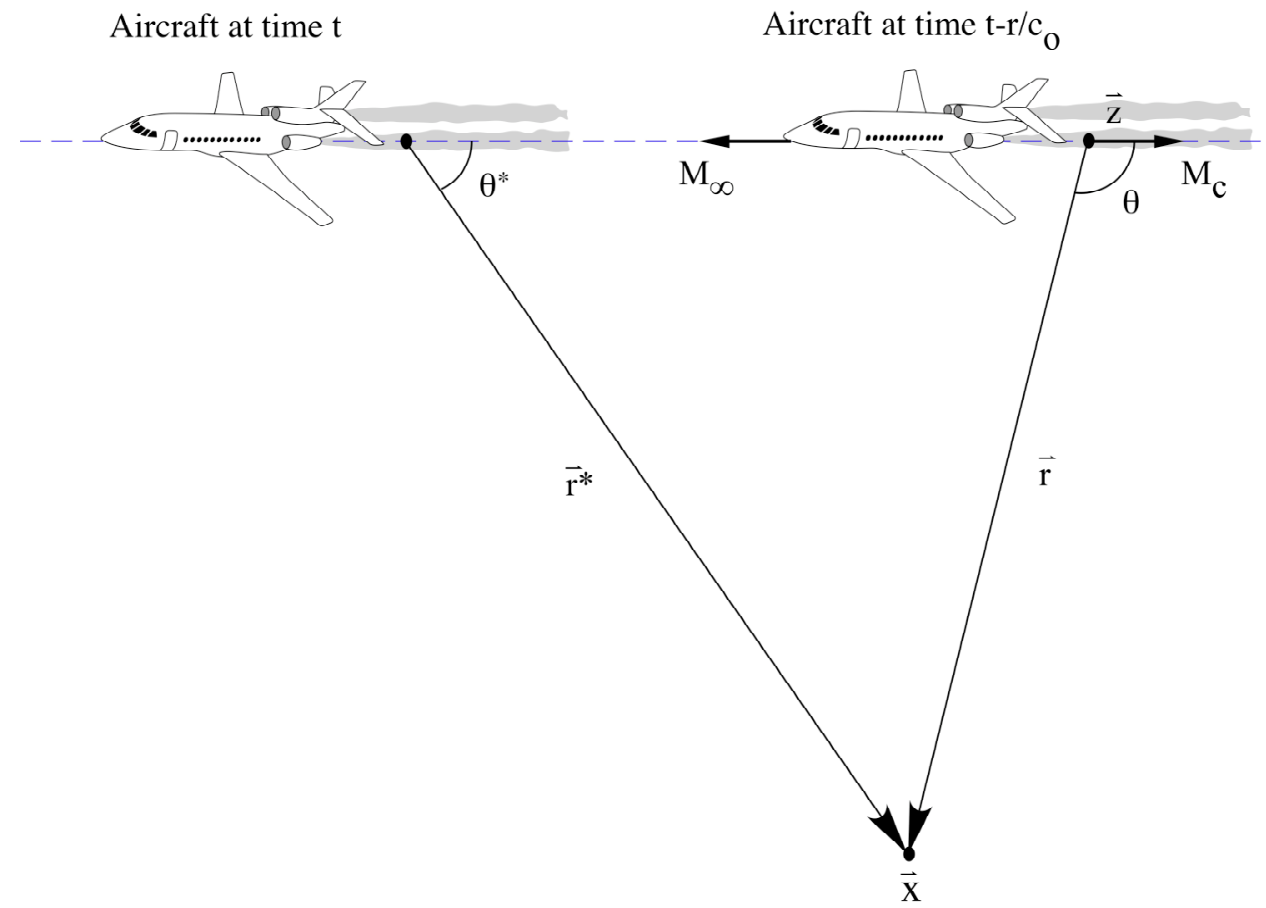

Observer at time $\mathrm{t}$

(fixed to ground)

Figure 1: Motion and Convection for the Lighthill/Ffowes Williams Acoustic Analogy Formulation.

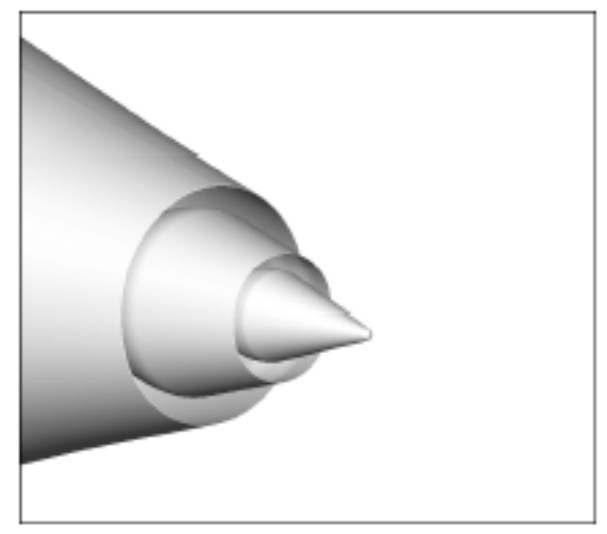

Figure 2: Baseline Round BPR Five Core/Fan Nozzle (Configuration 1). 
SPL noise prediction results with and without the retarded time correction are shown in Figures 3-5 at inlet angles of $55^{\circ}, 88^{\circ}$, and $121^{\circ}$, compared to experimental $100 \mathrm{D}_{\mathrm{c}}$ circular-arc acoustic data obtained at the NASA Langley Low Speed Aeroacoustic Wind Tunnel (LSAWT).

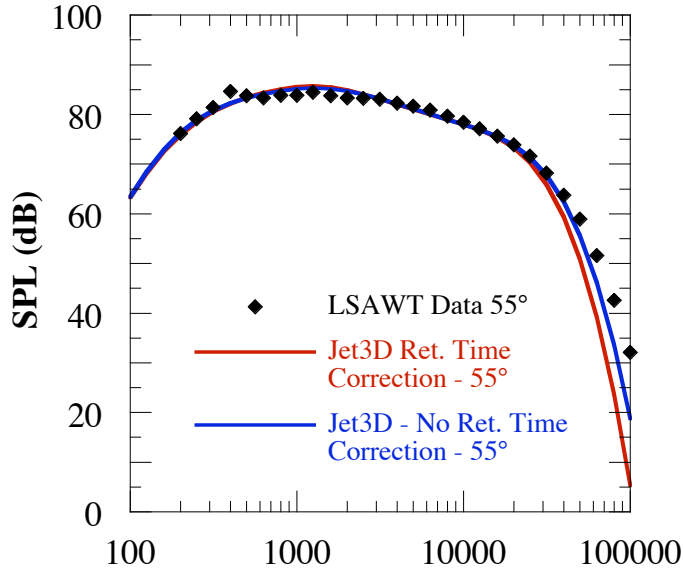

1/3 Octave Band Center Frequency $(\mathrm{Hz})$

Figure 3: SPL Predictions Compared to Experimental Data for an Inlet Angle of 55․

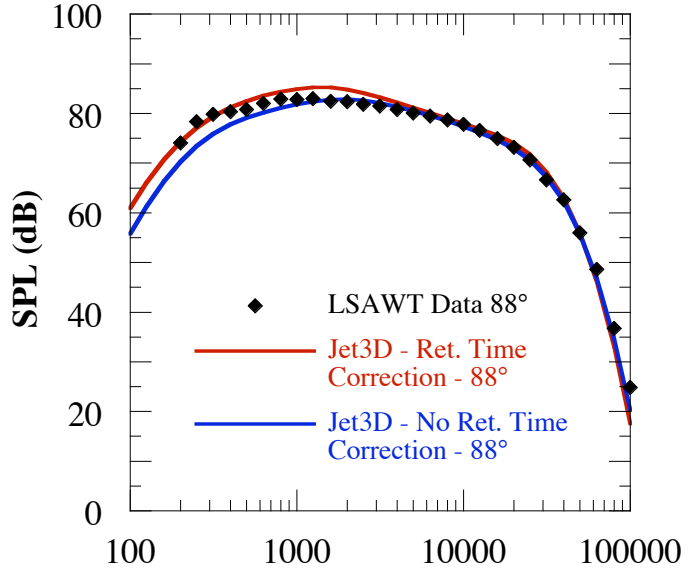

1/3 Octave Band Center Frequency $(\mathrm{Hz})$

Figure 4: SPL Predictions Compared to Experimental Data for an Inlet Angle of 88 ${ }^{\circ}$.

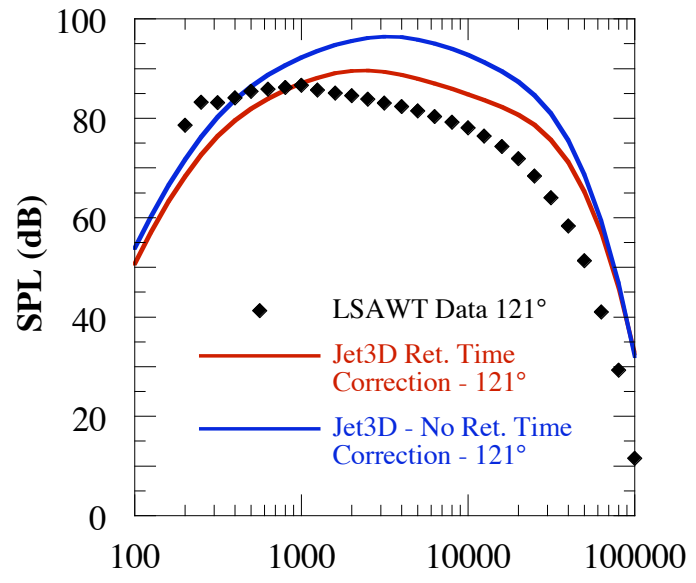

1/3 Octave Band Center Frequency $(\mathrm{Hz})$

Figure 5: SPL Predictions Compared to Experimental Data for an Inlet Angle of $121^{\circ}$.

The retarded time correction narrows the spectrum slightly at $55^{\circ}$ for greater high frequency under-prediction, but widens the spectrum at $88^{\circ}$ for better agreement with experimental data. While the spectral shape at $121^{\circ}$ is not correct with either prediction, the retarded time correction lowers the peak SPL by about $8 \mathrm{~dB}$, significantly reducing aft-arc over-prediction (thought to be due to incomplete modeling of the Lighthill Stress Tensor). 


\section{Application of Experimental Results}

As previously discussed, the accuracy of noise predictions using the Acoustic Analogy is closely dependent upon the accurate characterization of sources in the Lighthill Stress Tensor. Although no measurement technique is yet quite powerful enough to provide time-accurate measurements of a volume of turbulence in a high-speed, heated, multi-stream flow, there have been many advances in laser-based measurements in recent decades. In the current work, Particle Image Velocimetry (PIV) measurements ${ }^{14}$ are used to evaluate turbulent statistics such as Reynolds stresses and spatial correlations. The PIV technique uses a double-pulsed laser light sheet to illuminate seed particles in a flow field of interest - in this case along the jet centerline as shown in Figure 6. Individual particles are tracked by cross-correlating two successive digital camera images separated in time by microseconds. The result is a two-component instantaneous velocity measurement in a plane of the jet. Numerous image pairs can then be ensemble-averaged to obtain mean and turbulent statistics.

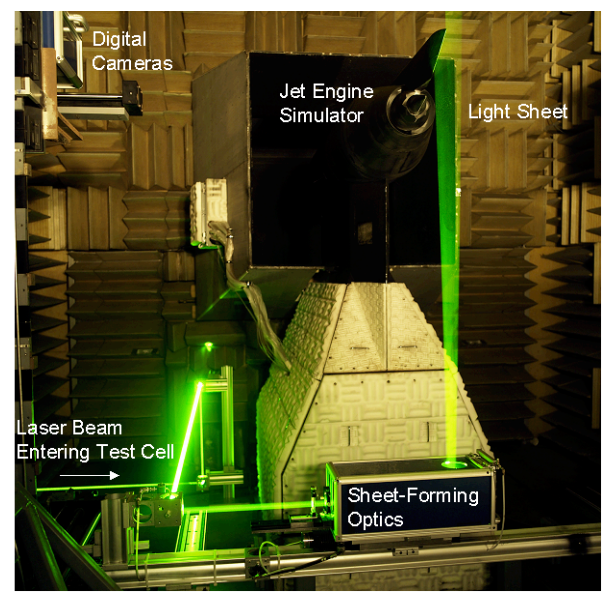

Figure 6: NASA Langley Low-Speed Aeroacoustic Wind Tunnel (LSAWT) with PIV set-up.

The turbulent flow fields of several BPR five nozzle configurations were measured using PIV. Two particular configurations are discussed in the current work: the baseline round core/fan nozzle (Configuration 1) previously shown in Figure 2 and a round core/fan nozzle with a pylon and lower fan bifurcator strut (Configuration 6), which is shown in Figure 7. Both nozzles were run at the takeoff conditions previously described in Section III.

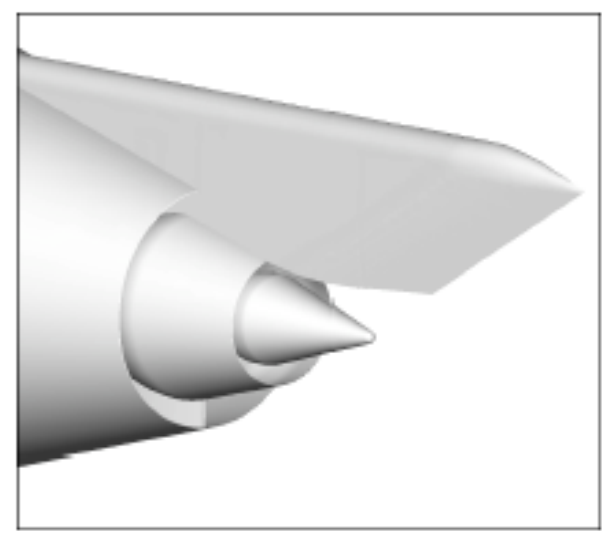

Figure 7: Nozzle Configuration 6. 


\section{A. Mean Flow and Reynolds Stress}

Successful noise prediction methods rely on accurate mean flow and turbulence computations. For consistent and accurate noise predictions, CFD computations need to be benchmarked against experimental measurements. Noise predictions presented in this paper are based on the computations of Massey et al. ${ }^{15}$, using the PAB3D RANS solver with temperature-corrected $k-\varepsilon$ turbulence closure and a linear Reynolds stress representation. Figures 8-11 show comparisons between the CFD predictions and the PIV measurements. Figure 8 shows close agreement between the mean axial velocity flow fields. The Reynolds shear stress value $\left\langle v_{1} v_{2}\right\rangle$ is slightly over-predicted by the CFD in Figure 9. However, the two normal components of Reynolds stress that the current PIV system is capable of measuring, $\left\langle v_{1} v_{1}\right\rangle$ and $\left\langle v_{2} v_{2}\right\rangle$, are significantly under-predicted by the CFD in Figures 10 and 11. Similar prediction trends were also noted in Configuration 1, which is not presented here due to asymmetry issues described in Doty et al. ${ }^{14}$ Work is ongoing to resolve the differences between the PIV and CFD results and to make improvements to the Reynolds stress predictions.
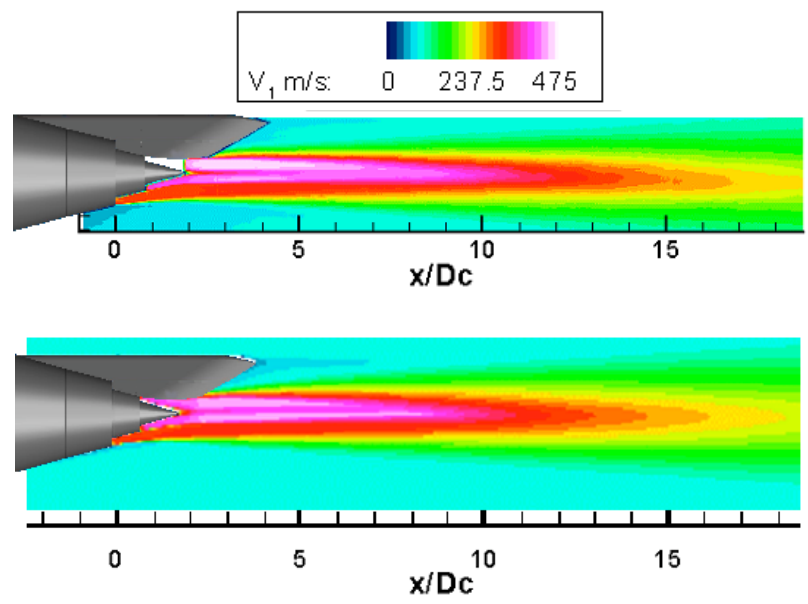

Figure 8: Comparison of $U$ for PIV (top) and CFD (bottom) for Configuration 6.

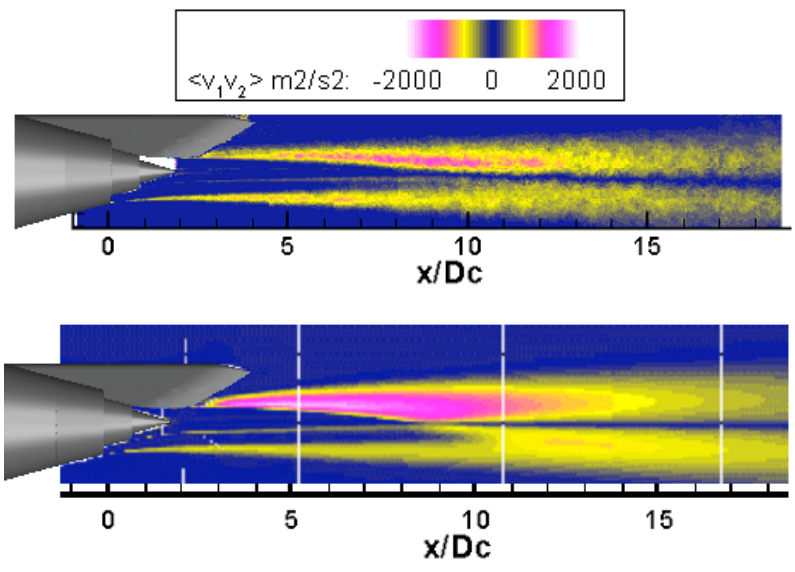

Figure 9: Comparison of $\left\langle v_{1} v_{2}>\right.$ for PIV (top) and CFD (bottom) for Configuration 6. 

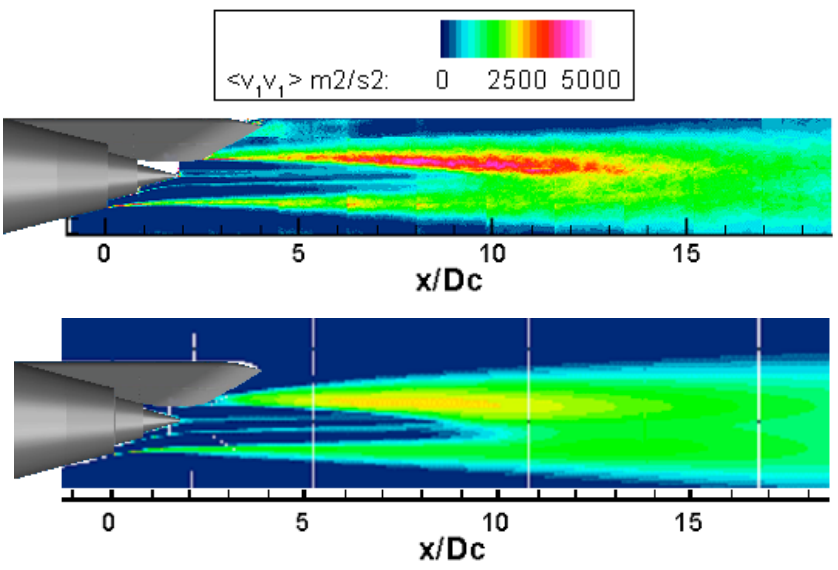

Figure 10: Comparison of $\left\langle v_{1} v_{1}\right\rangle$ for PIV (top) and CFD (bottom) for Configuration 6.
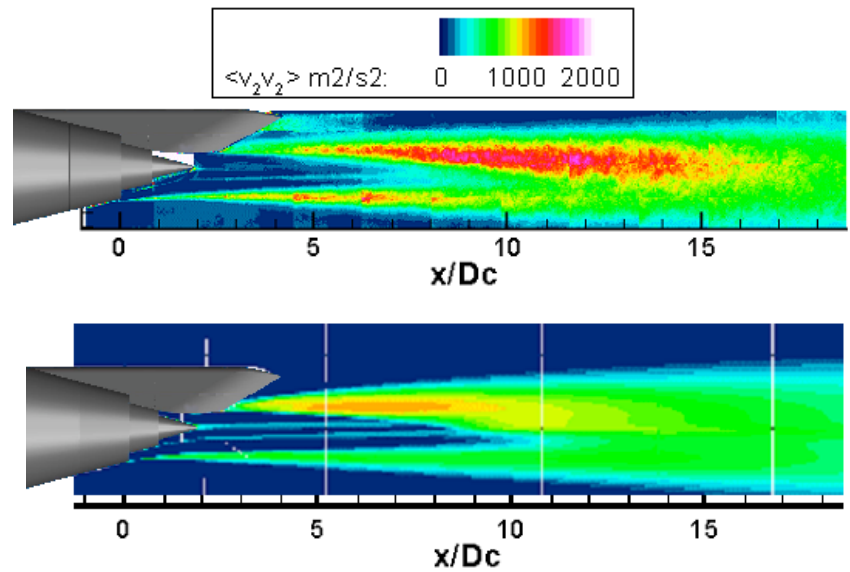

Figure 11: Comparison of $\left\langle v_{2} v_{2}>\right.$ for PIV (top) and CFD (bottom) for Configuration 6.

\section{B. Spatial Correlations}

Experimental efforts to characterize the Lighthill stress tensor by measuring two-point turbulent velocity correlations have been underway for some time. Davies et al. ${ }^{16}$, Bradshaw et al. ${ }^{17}, \mathrm{Chu}^{18}$, Harper Bourne ${ }^{19}$, Bridges and Podboy ${ }^{20}$, and others have contributed two-point correlation measurements using hot-wire instrumentation. However, the focus of these studies was limited to lower Mach number flows (typically $M=0.7$ and below) and cold jets due to the many difficulties of using hot-wires in higher Mach number heated flows. Crossed beam laser measurements ${ }^{21-23}$, deflectometry methods ${ }^{24,25}$, and Laser Doppler Velocimetry measurements ${ }^{26}$ have extended correlation measurements to higher Mach number, and in some cases heated, jet flow fields. More recently, Bridges and Wernet ${ }^{27}$ have obtained spatial correlations in high-speed heated jets using a single PIV set-up and space-time correlations using an impressive set-up of two PIV systems ${ }^{28}$. Note that a single PIV system has a limited frequency response on the order of 10-100 $\mathrm{Hz}$ based on laser repetition rate and digital camera acquisition rates. Thus, timeresolved correlations of high-speed jets are not typically possible with a single PIV system. The current work describes spatial correlations obtained from PIV measurements of Configuration 1 and subsequent comparisons to the model function used by Hunter ${ }^{13}$ in the Jet3D noise prediction code.

In a method similar to Bridges and Wernet ${ }^{27}$, spatial correlations are taken from a reference point defined as the center of the PIV image. A separation distance $(\xi \approx 4.3 \mathrm{~mm})$ corresponding to twice the resolution of the PIV data is selected, and the grid point spacing is $\xi / 2$ as shown in Figure 12. 


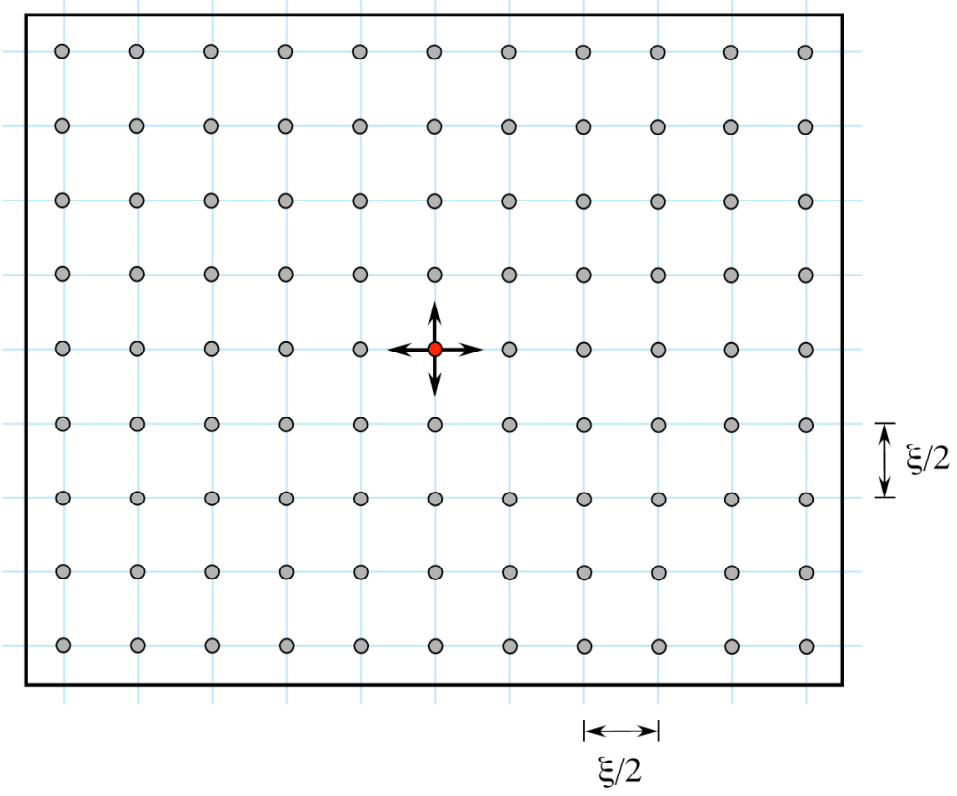

Figure 12: Map of Spatial Correlation Calculations.

Correlations are calculated by marching out from the reference (center) point to approximately thirty points on either side. The instantaneous velocities are multiplied, and averaged over the number of images taken (typically 400800). The correlation coefficient is then normalized by the mean-square value at the reference point to form the correlation coefficient function:

$$
\rho_{i j}=\frac{\left\langle v_{i}\left(\vec{x}+\frac{\vec{\xi}_{i}}{2}\right) * v_{j}\left(\vec{x}-\frac{\vec{\xi}_{i}}{2}\right)\right\rangle}{\sqrt{\left\langle v_{i}^{2}\right\rangle *\left\langle v_{j}^{2}\right\rangle}} .
$$

A correlation curve is generated by plotting $\rho_{i j}$ versus separation distance, and the integral length scale is calculated according to:

$$
L_{i k}(\vec{x})=\int_{0}^{\infty} \rho_{i i}\left(\xi_{k}, \vec{x}\right) d \xi_{k} .
$$

In this case, the integration is evaluated numerically using the trapezoidal rule. Because the spatial correlation is limited to the physical size of the PIV image (approximately $12 \mathrm{~cm}$ ), the integrals may be truncated prematurely in cases where the correlation coefficient function has not yet decayed to zero by the edges of the image.

Figure 13 shows a typical spatial correlation curve at a reference point of $x / D_{c}=15.5$ and $y / D_{c}=-0.32$. Also plotted is the two-point correlation "Model 1" currently used by Hunter ${ }^{13}$ in Jet3D:

$$
R_{\text {im }}(\vec{z}, \vec{\zeta})=<v_{i} v_{m}>\left[1-\frac{1}{\mu^{2}}\left(\frac{\varsigma_{1}^{2}}{l_{1}^{2}}+\frac{\varsigma_{2}^{2}}{l_{2}^{2}}+\frac{\varsigma_{3}^{2}}{l_{3}^{2}}\right)\right] \exp \left\{-\pi\left(\frac{\varsigma_{1}^{2}}{l_{1}^{2}}+\frac{\varsigma_{2}^{2}}{l_{2}^{2}}+\frac{\varsigma_{3}^{2}}{l_{3}^{2}}\right)\right\},
$$

where the directional turbulence length scale, $l_{i}$, is computed from RANS-CFD turbulence results:

$$
l_{i}=\alpha_{L} \frac{<v_{i}^{2}>^{3 / 2}}{\varepsilon} .
$$




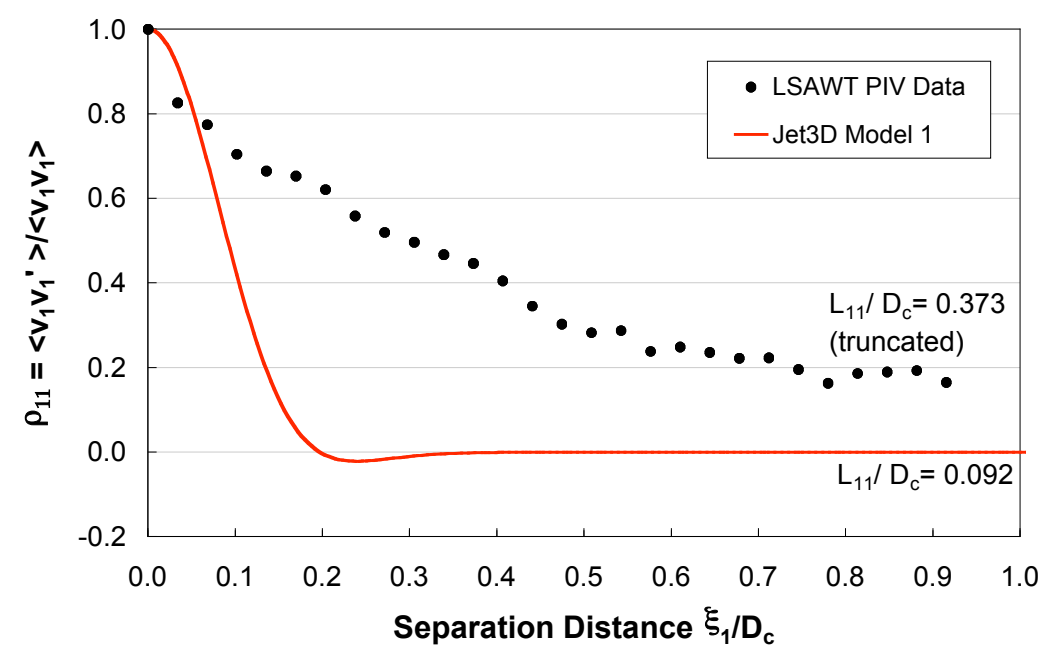

Figure 13: $\rho_{11}$ Comparisons at $x / D_{c}=15.5, y / D_{c}=-0.32$.

The disparity between the experiments and the Jet3D correlation model is significant. The integral length scale calculated from the PIV data is about a factor of four larger than the Jet3D integral scale, which is currently calibrated, using the $\alpha_{L}$ coefficient, to best match acoustic data. The shape of the correlation is also different. The variation of length scale with downstream axial distance at $y / D_{c}=0.57$ is shown in Figure 14. Keep in mind that the length scales far from the nozzle exit are actually higher than what the PIV measurements represent, due to the truncation of data at the edges of the image. Again, it is apparent that the Jet3D length scales are lower than those determined from the PIV data. Efforts are underway to re-evaluate the Jet3D length scale and investigate the discrepancy with PIV. The remainder of this work, however, will focus on the shape of the correlation curve and its effects on acoustic predictions.

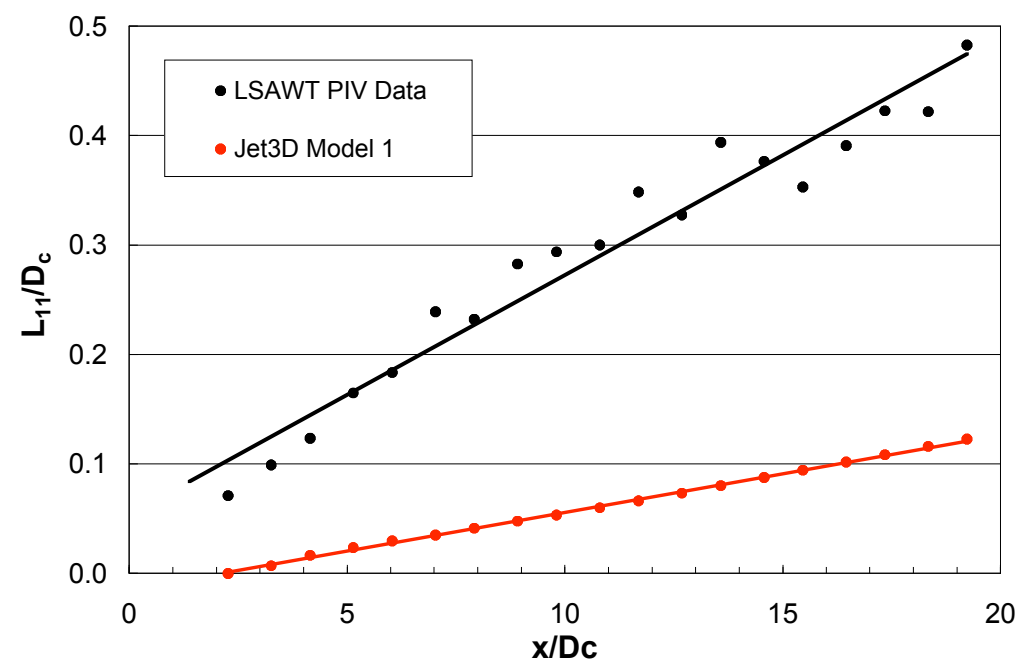

Figure 14: Nondimensional Length Scale Comparison at $y / D_{c}=0.57$.

In Figure 15, PIV correlation data at $x / D_{c}=15.5$ and $y / D_{c}=-0.32$ are shown along with the Jet3D correlation Model 1 and an exponential decay. Both models were computed for an integral scale of $\mathrm{L}_{11} / \mathrm{D}_{\mathrm{c}}=0.043$ such that a direct qualitative comparison of the correlation functions can be made. As Bridges and $\mathrm{Wernet}^{27}$ pointed out, 
exponential decay seems more appropriate than the Gaussian distribution currently used in most models, including Jet3D. For instance, a model of the form:

$$
R_{i m}(\vec{z}, \vec{\zeta})=<v_{i} v_{m}>\exp \left\{-\left(\left|\frac{\varsigma_{1}}{l_{1}}\right|+\left|\frac{\varsigma_{2}}{l_{2}}\right|+\left|\frac{\varsigma_{3}}{l_{3}}\right|\right)\right\}
$$

seems a more appropriate fit of the data as shown in Figure 15.

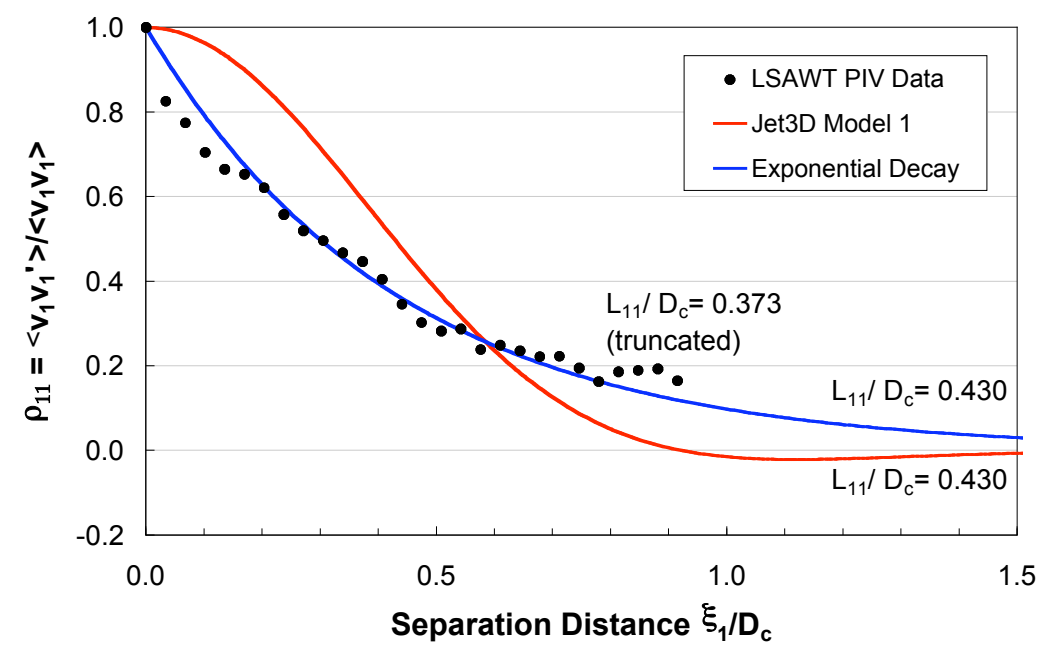

Figure 15: $\rho_{11}$ Comparisons at $x / D_{c}=15.5, y / D_{c}=-0.32$.

The exponential model in equation 9 was implemented in Jet3D as "Model 4". Predicted SPL results using this model are compared to results using Jet3D correlation Model 1 at inlet angles of $55^{\circ}, 88^{\circ}$, and $121^{\circ}$ in Figures 16 18. In both cases, the models have been calibrated for the best agreement with data at both $55^{\circ}$ and $88^{\circ}$. While the exponential model provides decent results, clearly they are not as good as the standard model at $55^{\circ}$ and $88^{\circ}$. One reason for this is the quadratic zero crossing component of Model 1 (see eq. 7), which results in an additional calibration constant $(\mu)$ that can weight shear and self noise contributions to shape the SPL spectrum.

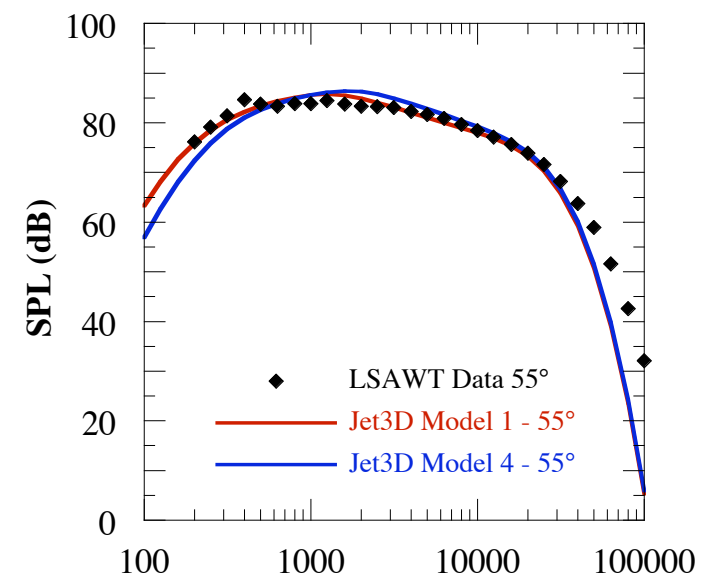

1/3 Octave Band Center Frequency (Hz)

Figure 16: Comparisons of Model $1(\mu=0.82$ $\left.\alpha_{L}=0.45\right)$ and Model $4\left(\alpha_{L}=0.11\right)$ at $55^{\circ}$.

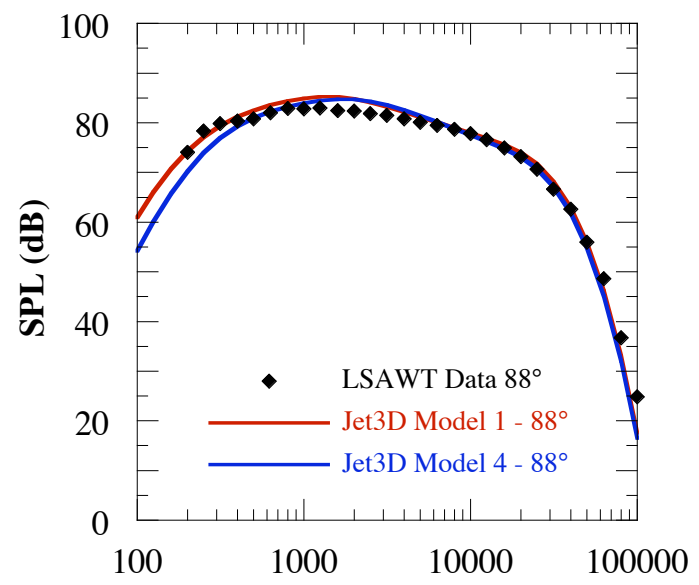

1/3 Octave Band Center Frequency $(\mathrm{Hz})$

Figure 17: Comparisons of Model $1(\mu=0.82$ $\left.\alpha_{L}=0.45\right)$ and Model $4\left(\alpha_{L}=0.11\right)$ at $88^{\circ}$. 


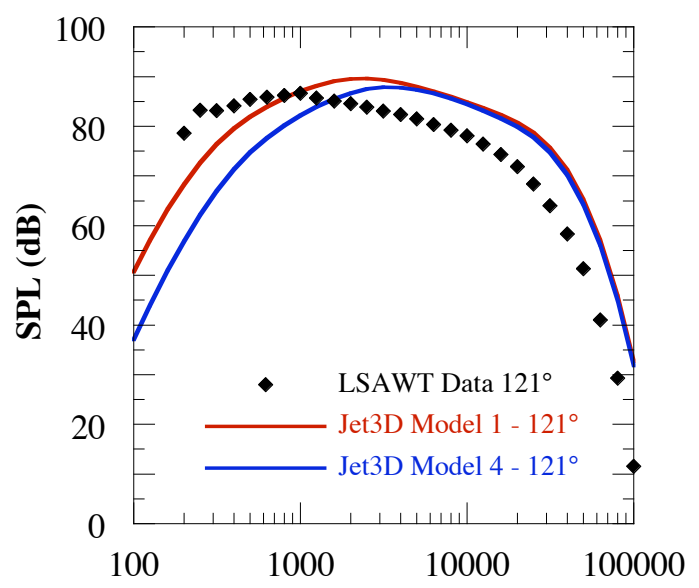

1/3 Octave Band Center Frequency $(\mathrm{Hz})$

Figure 18: Comparisons of Model $1\left(\mu=0.82 \alpha_{L}=0.45\right)$ and Model $4\left(\alpha_{L}=0.11\right)$ at $121^{\circ}$.

To explore this point, a quadratic zero crossing component was added to the exponential model in equation 9 , resulting in Model 5:

$$
R_{i m}(\vec{z}, \vec{\zeta})=<v_{i} v_{m}>\left[1-\frac{1}{\mu^{2}}\left(\frac{\varsigma_{1}^{2}}{l_{1}^{2}}+\frac{\varsigma_{2}^{2}}{l_{2}^{2}}+\frac{\varsigma_{3}^{2}}{l_{3}^{2}}\right)\right] \exp \left\{-\left(\left|\frac{\varsigma_{1}}{l_{1}}\right|+\left|\frac{\varsigma_{2}}{l_{2}}\right|+\left|\frac{\varsigma_{3}}{l_{3}}\right|\right)\right\} .
$$

Results from Model 5 are compared to Model 1 in Figures 19-21, and the two models are indistinguishable (note that the models were calibrated independently). This may seem surprising since one model is Gaussian and the other is exponential; however, evaluating the spatial integral of both correlation models nets similar results:

$$
\begin{gathered}
\text { Model } 1 \text { with } \boldsymbol{\mu}=\mathbf{0 . 8 2} \text { and } \boldsymbol{\alpha}_{\mathrm{L}}=\mathbf{0 . 4 5} \\
\int_{-\infty}^{\infty}\left[1-\frac{1}{\mu^{2}}\left(\frac{\varsigma_{1}^{2}}{l_{1}^{2}}+\frac{\varsigma_{2}^{2}}{l_{2}^{2}}+\frac{\varsigma_{3}^{2}}{l_{3}^{2}}\right)\right] \exp \left\{-\pi\left(\frac{\varsigma_{1}^{2}}{l_{1}^{2}}+\frac{\varsigma_{2}^{2}}{l_{2}^{2}}+\frac{\varsigma_{3}^{2}}{l_{3}^{2}}\right)\right\} d \vec{\zeta}=\frac{2 \pi\left(2 \pi \mu^{2}-3\right) l_{1} l_{2} l_{3}}{4 \pi^{2} \mu^{2}}=0.0264\left(\frac{\left.\left.\left\langle v_{1}^{2}\right\rangle^{3 / 2}<v_{2}^{2}\right\rangle^{3 / 2}<v_{3}^{2}\right\rangle^{3 / 2}}{\varepsilon^{3}}\right),
\end{gathered}
$$

Model 5 with $\mu=2.65$ and $\alpha_{L}=0.28$

$$
\int_{-\infty}^{\infty}\left[1-\frac{1}{\mu^{2}}\left(\frac{\varsigma_{1}^{2}}{l_{1}^{2}}+\frac{\varsigma_{2}^{2}}{l_{2}^{2}}+\frac{\varsigma_{3}^{2}}{l_{3}^{2}}\right)\right] \exp \left\{-\left(\left|\frac{\varsigma_{1}}{l_{1}}\right|+\left|\frac{\varsigma_{2}}{l_{2}}\right|+\left|\frac{\varsigma_{3}}{l_{3}}\right|\right)\right\} d \vec{\zeta}=\frac{8\left(\mu^{2}-6\right) l_{1} l_{2} l_{3}}{\mu^{2}}=0.0256\left(\frac{<v_{1}^{2}>^{3 / 2}<v_{2}^{2}>^{3 / 2}<v_{3}^{2}>^{3 / 2}}{\varepsilon^{3}}\right) \text {. }
$$

This suggests that the mathematical form of the correlation model is not so important as long as the function is integrable. Moreover, this suggests that skipping the correlation integral altogether and proposing generic models of the form $l_{1} l_{2} l_{3}$ (with suitable calibration constants) is every bit as suitable as the current approach. 


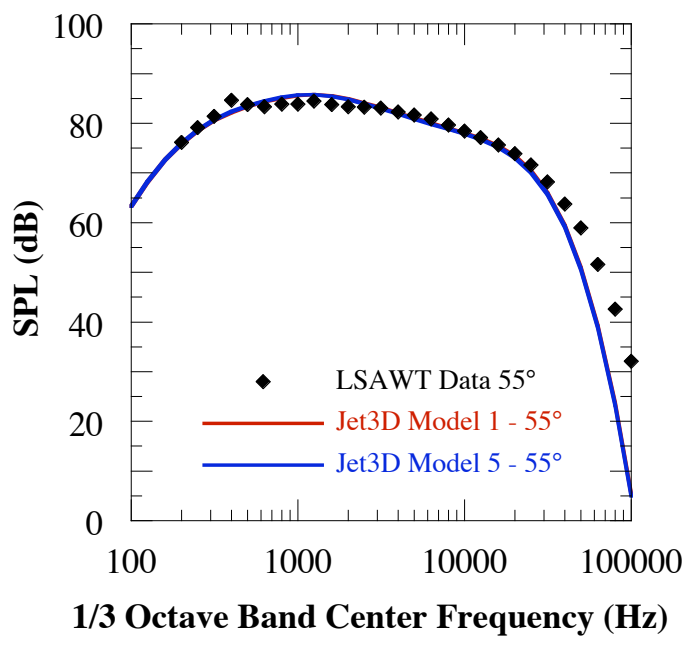

Figure 19: Comparisons of Model $1(\mu=0.82$ $\left.\alpha_{L}=0.45\right)$ and Model $5\left(\mu=2.65 \alpha_{L}=0.28\right)$ at $55^{\circ}$.

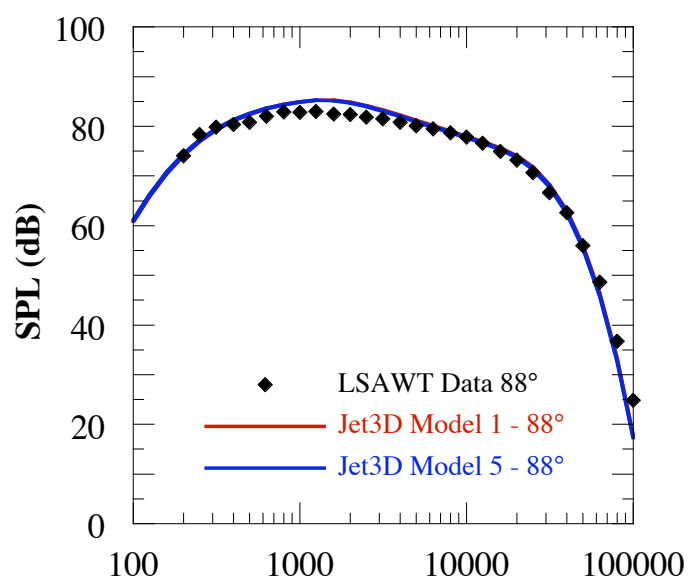

1/3 Octave Band Center Frequency $(\mathrm{Hz})$
Figure 20: Comparisons of Model $1(\mu=0.82$ $\left.\alpha_{\mathrm{L}}=0.45\right)$ and Model $5\left(\mu=2.65 \alpha_{\mathrm{L}}=0.28\right)$ at $88^{\circ}$.

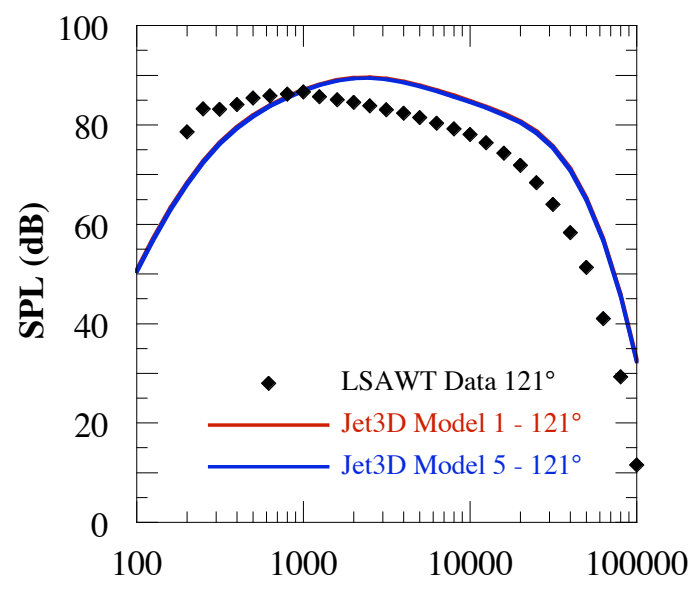

1/3 Octave Band Center Frequency (Hz)

Figure 21: Comparisons of Model $1\left(\mu=0.82 \alpha_{L}=0.45\right)$ and Model $5\left(\mu=2.65 \alpha_{L}=0.28\right)$ at $121^{\circ}$.

\section{Concluding Remarks}

This paper discusses Lighthill's Acoustic Analogy and the associated acoustic sources in general terms. Specific application of the technique to jet noise prediction is examined in detail with collaboration among analytical, computational and experimental backgrounds. A retarded time correction was developed that improves aft-arc noise prediction by some $8 \mathrm{~dB}$ at an inlet angle of $121^{\circ}$ using the Jet3D prediction code. PIV measurements have provided a basis for comparison of CFD Reynolds stresses as well as two-point correlation modeling used in Jet3D. Experimental mean flow field and Reynolds shear stresses are in reasonable agreement with CFD, with slight overprediction of $\left\langle v_{1} v_{2}\right\rangle$. The Reynolds normal stresses, however, are significantly under-predicted by CFD. Initial investigations of experimental two-point spatial correlation data seem to indicate the need for improvements to the correlation model used in Jet3D, both in functional form and in model length scale. However, changing the 
correlation model in Jet3D from a Gaussian to an exponential form suggested by the PIV results made no significant changes in the acoustic predictions. This is due to the fact that both the exponential and Gaussian models give the same analytical result (differing only by calibration constants) when correlation integrals are evaluated. The discrepancy in integral length scales between PIV and CFD, as well as the inability to fully match acoustic data in the aft arc of the jet, indicate the potential for further modeling improvements including a more complete model of the Lighthill Stress Tensor within Jet3D.

\section{Acknowledgments}

The authors thank Aeroacoustics Branch Head, Joe Posey, for support, as well as the PAA CFD team for their contributions to this work: S. Paul Pao, Steven Massey, Alaa Elmiligui, and K.S. Abdol-Hamid. M. J. Doty also wishes to acknowledge the National Research Council for program administration during this work.

\section{References}

1 Lighthill, M. J., "On Sound Generated Aerodynamically: I. General Theory”, Proceeding of the Royal Society of London, Series A Volume 211, 1952, pp. 564-587.

2 H. S. Ribner, "The Generation of Sound by Turbulent Jets," Advances in Applied Mechanics, Vol. 8, 1964, pp. 104-182.

3 Lilley, G. M., "On the Noise from Air Jets,” Aeronautical Research Council Report ARC-20376, 1958.

4 Curle, N., "The Influence of Solid Boundaries upon Aerodynamic Sound," Proceedings of the Royal Society of London, Vol. A231, 1955, pp. 505-514.

5 Ffowcs Williams, J. E., "The Noise from Turbulence Convected at High Speed," Philosophical Transactions of the Royal Society of London, Series A, Vol. 255, 1963, pp. 469-503.

6 Ffowcs Williams, J. E. and Hawkings D.L., "Sound Generation by Turbulence and Surfaces in Arbitrary Motion," Philosophical Transactions of the Royal Society of London, Series A, Vol. 264, 1969, pp. 321-342.

7 Personal communication between G. M. Lilley and C.A. Hunter, March 6, 2004.

8 Farassat, F. and Brentner, K. S., "Supersonic Quadrupole Noise Theory for High-Speed Helicopter Rotors," Journal of Sound and Vibration, Vol. 218(3), 1998, pp. 481-500.

9 Girimaji, S.S. "Partially Averaged Navier-Stokes (PANS) Approach: A RANS to LES Bridging Model”. The American Physical Society 56th Annual Meeting of the Division of Fluid Dynamics, November 23-25, 2003.

10 Brentner, K. S. and Farassat, F., "Modeling Aerodynamically Generated Sound of Helicopter Rotors," Progress in Aerospace Sciences, Vol. 39, 2003, pp. 83-120.

${ }^{11}$ Farassat, F. and Casper, J., "Some Analytic Results for the Study of Broadband Noise Radiation from Wings, Propellers and Jets in Uniform Motion," International J. of Aeroacoustics, Vol. 2 - number 3 \& 4, 2003, pp. $333-348$.

12 Hunter, C., "An Approximate Jet Noise Prediction Method Based on Reynolds-Averaged Navier-Stokes Computational Fluid Dynamics Simulation ," PhD Thesis, Mechanical Engineering Dept., The Pennsylvania State University, University Park, PA, 1994.

13 Hunter, C. and Thomas, R., "Development of a Jet Noise Prediction Method for Installed Jet Configurations," AIAA Paper 2003-3169, May 2003. 
Doty, M. J., Henderson, B. S., and Kinzie, K. W., "Turbulent Flow Field Measurements of Separate Flow Round and Chevron Nozzles with Pylon Interaction Using Particle Image Velocimetry,” AIAA Paper 2004-2826, May 2004.

15 Massey, S.J., Thomas, R.H., Abdol-Hamid, K.S. and Elmiligui, A.A., "Computational and Experimental Flowfield Analyses of Separate Flow Chevron Nozzles and Pylon Interaction," AIAA Paper 2003-3212, May 2003.

16 Davies, P. O. A. L., Fisher, M. J., and Barratt, M. J., "The Characteristics of the Turbulence in the Mixing Region of a Round Jet," Journal of Fluid Mechanics, Vol. 15, 1962 pp. 337-367.

17 Bradshaw, P., Ferriss, D. H., and Johnson, R. F., "Turbulence in the Noise-Producing Region of a Circular Jet," Journal of Fluid Mechanics, Vol. 19, 1963, pp. 591-624.

18 Chu, Wing T., “Turbulence Measurements Relevant to Jet Noise,” UTIAS Report No. 119, November 1966.

19 Harper-Bourne, M., “Jet Near-Field Noise Prediction,” AIAA Paper 99-27825.

20 Bridges, J. and Podboy, G.G., "Measurements of Two-Point Velocity Correlations in a Round Jet with Application to Jet Noise," AIAA Paper 99-1966, 1999.

21 Funk, B. H., and Johnston, K. D., "Laser Schlieren Crossed-Beam Measurements in a Supersonic Jet Shear Layer," AIAA Journal, Vol. 8, No. 11, 1970, pp. 2074, 2075.

${ }^{22}$ Harper-Bourne, M. and Fisher, M. J., "The Noise from Shock Waves in Supersonic Jets," Noise Mechanisms, AGARD-CP-131, 1974, pp. 11.1-11.13.

${ }^{23}$ Damkevala, R. J., Grosche, F. R., and Guest, S. H., "Direct Measurement of Sound Sources in Air Jets Using the Crossed Beam Correlation Technique," Noise Mechanisms, AGARD-CP-131, 1974, pp. 3.1-3.16.

24 McIntyre, S., "Optical Experiments on Axisymmetric Compressible Mixing Layers," PhD Thesis, Mechanical Engineering Dept., The Pennsylvania State University, University Park, PA, 1994.

25 Doty, M. J. and McLaughlin, D. K., "Two-Point Correlations of Density Gradient Fluctuations in High Speed Jets Using Optical Deflectometry,” AIAA Paper 2002-0367, January 2002.

26 Lau, J. C., "Laser Velocimeter Correlation Measurements in Subsonic and Supersonic Jets," Journal of Sound and Vibration, Vol. 70, 1980, pp. 85-101.

27 Bridges, J. and Wernet, M. P., "Turbulence Measurements of Separate Flow Nozzles with Mixing Enhancement Features," AIAA Paper 2002-2484, June 2002.

28 Bridges, J. and Wernet, M. P., "Measurements of the Aeroacoustic Sound Source in Hot Jets," AIAA Paper 2003-3130, May 2003. 